\title{
Variance Analysis-Based Chinese Urban and Rural Sports Development and Urbanization Construction Correlation Research
}

\author{
Shengguo Wang ${ }^{1, *}$, Jinjuan Wang ${ }^{2}$ and $\mathrm{Li} \mathrm{Lin}^{3}$ \\ ${ }^{1}$ Department of Physical Education, DaLian University of Foreign Languages, DaLian 116044, Liaoning, China; \\ ${ }^{2}$ Institute of Physical Education, Liaoning Normal University, DaLian 116029, Liaoning, China; ${ }^{3}$ Department of \\ Physical Education, MinJiang University, Fuzhou 350108, Fujian, China
}

\begin{abstract}
With Chinese economic rapidly development, urban and rural difference are also increasing, no matter in living standards, living conditions or spiritual life pursuit, cultural quality, all have obvious differences. It also causes urban and rural sports to have bigger differences in development process. The paper researches on Chinese urban and rural sports development basic status, makes concrete analysis of urban and rural sports differences in residents' sports activities participation forms, urban and rural sport interactive organizations, urban and rural residents sports consciousness and urban and rural residents sports satisfaction index and other aspects. Utilize variance analysis and correlation analysis, establish relevant variance analysis-based urban and rural sports development and urbanization construction model, finally it gets conclusions: Chinese urban sports development is faster with respect to rural sports, the two have significant differences in every aspect construction. Urban and rural sports relations are closely, inseparable, the two mutual restrict, jointly develop, only well develop rural sports then can better carry out urban sports construction, and then can really propel to new pattern urbanization construction development to make contributions to urbanization development.
\end{abstract}

Keywords: Correlation analysis, urban and rural sports, urbanization construction, variance analysis.

\section{INTRODUCTION}

Gap between urban and rural districts are one of important problems that always trouble China development, shorten urban and rural gap, speed up urban and rural collaboration, integration development, which have been listed into top priority in Chinese development. And urban and rural sports is an important part of urban and rural development, in order to research on urban and rural sports development gap, and its important roles in urbanization construction process, discover rural sports development process existing problems, shorten urban and rural sports, many scholars have made research and got excellent achievements [1].

Xiong Xue-Mei in the article "Research on Chinese urban and rural sports out-of-balance development status", found that current society China investment on urban and rural sports had considerable big differences, and put forward suggestions on how to reduce these differences [2]. The paper under the perspective of current fast pace of lifestyle, researched Chinese urban and rural sports.

The paper under the perspective of current fast pace of lifestyle, researched Chinese urban and rural sports development trend in current environment, and went on practical investigation, data processing, and finally got conclusions: resource distribution, family education status and others were origins that led to urban and rural imbalanced development, on this basis, proposed that sports resources should reasonable distribute, and meanwhile it should firstly start from school and provide important theoretical references for Chinese urban and rural sports development [3].

Xue Ling in the article "Urban and rural masses sports overall development exploration", took urban and rural economy and sports culture integration development strategy as main research objects, proposed that in contemporary, economy was rapidly developing, sports importance to China development and suggestions on how to promote people's emphasis on urban and rural sports [4]. The paper through interviewing schools, collecting urban and rural residents satisfaction index on current phase urban and rural sports, as well as researches on government macro-adjustment, current phase Chinese sports system and institutions, sports resources, finally put forward: it not only should adjust and control through government such intangible hand, but also should spread sports importance to contemporary residents' health by education then could propel to current Chinese urban and rural sports development undertakings [5].

Zhang Hong-Wei in the article "Urban and rural sports integrative development value dimension and practical guarantee", under urban and rural construction harmonious stable developing social environment, he researched on economy and sports culture harmonious and sustainable development. The paper through utilizing analysis and investigation research method, researched on contemporary urban and rural sports integrative development, and then propose that it should stepwise establish urban and rural sports system and institutions, strengthen sports administrative management strength so that arrive at realistic urban and rural sports culture and economy integration [6]. 
The paper utilizes mathematics variance analysis and correlation analysis, established mathematical model, and references lots of information, by software handling with data, researches on Chinese urban and rural sports development problem, reduces urban and rural sports development differences, and contributes ideas and exerts efforts for Chinese urbanization construction.

\section{URBAN AND RURAL SPORTS DEVELOPMENT COMPARISON}

Research on urban and rural sports development gap, it mainly analyzes the two gap sizes by analyzing urban and rural residents satisfaction index on sports, urban and rural residents sports consciousness, urban and rural residents sports participation status, urban and rural sports activities organizations frequency number. Therefore, utilize mathematics variance analysis method, handle with data, and analyze its differences' significance (YAN De-yi, 2006) [7].

\subsection{Urban and Rural Residents' Sports Participation Status}

Network, library, stadiums, clubs, friends, colleagues and others are main paths that public participate in sports. In network, it can spread sports through network sports games live broadcasting, sports news, sports video and other new pattern sports medias; in library, it mainly introduces sports culture by books and words; in sports clubs, it mainly encourages masses to participate in sports in the form of amateur sports activities; and during chatting process with friends and colleagues, which are also basic paths to spread sports culture, learning sports dynamics.
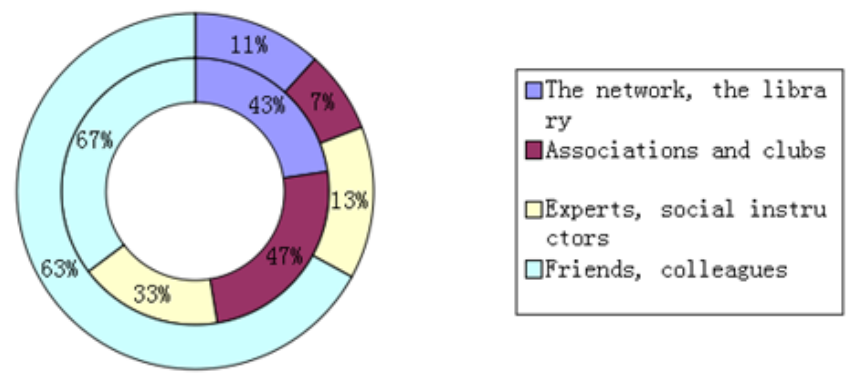

Fig. (1). The way to get the fitness education of urban and rural residents.

Above statistical Fig. (1) shows: For urban and rural residents, in the four kinds of sports spread paths applying process, due to urban residents' living standards, living state multiple aspects influences, its application has obvious gap.
Urban residents mainly participate in sports through network, library, stadium, clubs and other paths, while rural districts due to conditions restriction, network , stadium construction are not perfect, facilities are not completely, some villages even don't have stadiums, main ways of them participating in sports are by TV, chatting with friends and others.

\subsection{Urban and Rural Sports Activities Organizations}

In Chinese social life, due to government sector, assoctiation of social organizations and social sponsorship supports on sports activities, it leads to sports activities organizing status to be also different. For people that have certain knowledge on sports fitness, and love sports fitness, participate in sports activities is a kind of joy of life, and also is an indispensible part in daily life.

From above statistical Fig. (2), it is clear that association of social organizations is main force when urban and rural sports organize sports activities, which has close relations with organizers sports consciousness, organizing ability, organizing positivity. By comparing, rural sports are inferior to urban ones in sports activities organization aspect, and organizing way is relative single, it mainly bases on social sponsorship. It restricts rural sports development to certain extent, and then widens distances between urban and rural sports construction, which let urban sports and rural sports gap to be larger, and meanwhile also let urbanization construction to be blocked.

\subsection{Urban and Rural Residents' Sports Consciousness}

Citizen sports consciousness is an important factor that restricts sports development, is also extremely crucial factor. Affected by education extent, cultural standards differences, urban and rural residents' difference in sports consciousness aspect are significant. Below figure is urban and rural residents' sports consciousness statistical analysis figure, data is from China's statistical yearbook, general administration of sport of China and internet relevant investigation reports, analyze statistical figure and then further get conclusions.

Analyze above Fig. (3), it is clear that no matter city or countryside, residents' sports consciousness is overall higher; most of people think that sports can promote physical quality, keep fit. But there are still fewer people mixed manual work with physical exercises, regard manual work as another kind of physical exercises. The case is particularly serious in rural sports, due to farm work is quite a lot, physical exercise time is fewer, residents' cultural standards are

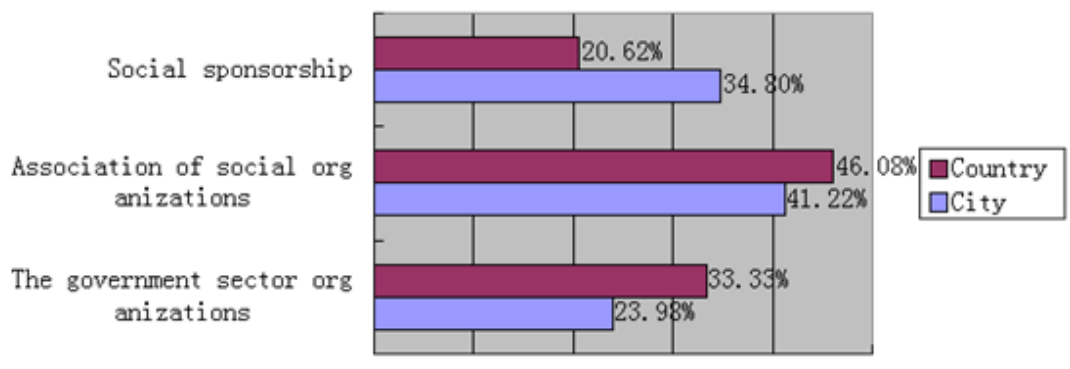

$0.00 \% \quad 10.00 \% \quad 20.00 \% \quad 30.00 \% \quad 40.00 \% \quad 50.00 \%$

Fig. (2). City and country sports organization. 


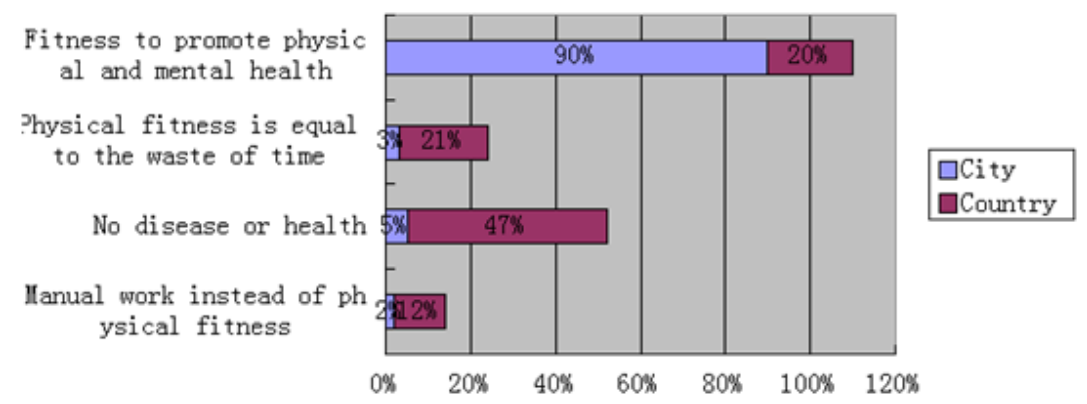

Fig. (3). The sport consciousness of urban and rural residents.

Table 1. Data statistical table.

\begin{tabular}{|c|c|c|c|}
\hline & Item & City & Countryside \\
\hline \multirow{4}{*}{ Sports forms participation } & The network, the library & 0.43 & 0.11 \\
\hline & Associations, clubs & 0.47 & 0.07 \\
\hline & Experts, social instructors & 0.33 & 0.13 \\
\hline & Friends, colleagues & 0.67 & 0.63 \\
\hline \multirow{3}{*}{ Sports activities organizations } & The government sector organizations & 0.2398 & 0.3333 \\
\hline & Associations of social organizations & 0.4122 & 0.4608 \\
\hline & Social sponsorship & 0.348 & 0.2062 \\
\hline \multirow{4}{*}{ Sports consciousness } & Manual work instead of physical fitness & 0.02 & 0.12 \\
\hline & No disease or health & 0.05 & 0.47 \\
\hline & Physical fitness is equal to the waste of time & 0.03 & 0.21 \\
\hline & Fitness to promote physical and mental health & 0.90 & 0.20 \\
\hline \multirow{4}{*}{ Public satisfaction index } & Satisfaction & 0.10 & 0.04 \\
\hline & Basic satisfaction & 0.40 & 0.16 \\
\hline & Dissatisfaction & 0.45 & 0.61 \\
\hline & Does not matter & 0.05 & 0.19 \\
\hline
\end{tabular}

relative lower, and then lead to rural residents' recognition degree on sports also to be inferior to urban residents.

\subsection{Urban and Rural Sports Public Satisfaction Index}

Chinese sports facilities construction development in urban and rural districts is not going well, especially for rural sports facilities construction. Affected by economic development, urban and rural sports facilities construction extents are also different, which affects urban and rural residents' satisfaction index on sports to certain extent. Below Fig. (4) is investigation comparison data of Chinese urban and rural residents satisfaction index on sports facilities, it is from China's statistical yearbook, general administration of sport of China and internet relevant investigation report.

From above statistical Fig. (4), it can analyze and get urban and rural residents sports satisfaction index differences are relative significant, urban residents satisfaction index is higher, which is benefit by urban sports construction relative perfection. And rural residents' satisfaction index is lower, which is mainly caused by rural sports development slowness. Therefore, perfect rural sports construction, develop rural sports, propel to urban and rural integration has become important tasks of current sports construction.

\section{URBAN AND RURAL SPORTS DEVELOPMENT DIFFERENCE MODEL UNDER VARIANCE ANALY- SIS}

For above kinds of urban and rural sports development intuitive evaluation factors, sort out its data into following Table 1, and utilize variance analysis method to carry out significance analysis of it.

\subsection{Two Factors Variance Analysis Guiding Thought}

Variance analysis is considering factors impacts on indicators. Research objects experiment result is indicator, control variable, condition that is factors. When research objects influence factors are two, it should consider two factors variance analysis. Set it has two influence factors 


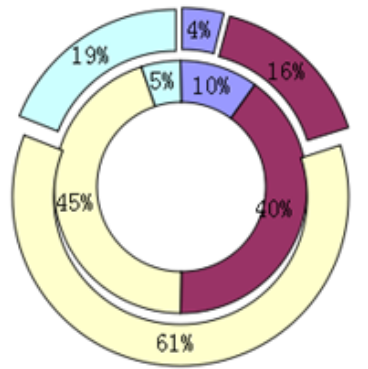

$\square$ Satisfaction

$\square$ Basic satisfaction $\square$ Dissatisfaction $\square$ Does not matter

Fig. (4). Urban and rural residents' sports facilities satisfaction.

$A, B$, make respective classification of $A, B$ into some levels, and make several times test on every level, make variance analysis of data, and then research on $A, B$ two influence factors respective significances on research objects impacts. Sometimes, it should make further test whether $A, B$ have significant interactive impacts on research objects.

Its mathematical model is: set $A$ to take $r$ pieces of level $A_{1}, A_{2}, \cdots, A_{r}, B$ takes s pieces of level $B_{1}, B_{2}, \cdots, B_{s}$, under level combination $\left(A_{i}, B_{j}\right.$ ), totality $x_{i j}$ conforms to normal distribution $N\left(\mu_{i j}, \delta^{2}\right), i=1, \cdots, r, j=1, \cdots, s$. And under $A_{i}, B_{j}$, it makes $t$ pieces of experiments, record result as $x_{i j k}, x_{i j k}$ conforms to $N\left(\mu_{i j}, \delta^{2}\right), i=1, \cdots, r, j=1, \cdots, s$, $k=1, \cdots, t$, and mutual independent. And then it can get following Table 2 .

Decompose $x_{i j k}$ into:

$x_{i j k}=\mu_{i j}+\varepsilon_{i j}, i=1, \cdots, r, j=1, \cdots, s, k=1, \cdots, t$

Among them, $\varepsilon_{i j k} \sim N\left(\mu_{i j}, \delta^{2}\right), \quad$ and mutual independent, record:

$$
\begin{aligned}
& \mu=\frac{1}{r s} \sum_{i=1}^{r} \sum_{j=1}^{s} \mu_{i j}, \mu_{i \bullet}=\frac{1}{s} \sum_{j=1}^{s} \mu_{i j}, a_{i}=\mu_{i \bullet}-\mu \\
& \mu_{i \bullet}=\frac{1}{r} \sum_{i=1}^{r} \mu_{i j}, \beta_{i}=\mu_{\bullet j}-\mu, \gamma_{i j}=\mu_{i j}-\mu-\alpha_{i}-\beta_{i}
\end{aligned}
$$

Among them, $\mu$ is grand average, $\alpha_{i}$ is level $A_{i}$ effect on indicator, $\beta_{i}$ is level $B_{i}$ effect on indicator, $\gamma_{i j}$ is level $A_{i}$ and level $B_{i}$ interaction effect on indicator. Model table is:

$$
\left\{\begin{array}{l}
x_{i j k}=\mu+\alpha_{i}+\beta_{j}+\gamma_{i j}+\varepsilon i_{j} \\
\sum_{i=1}^{r} \alpha_{i}=0, \sum_{j=1}^{s} \beta_{j}=0, \sum_{i=1}^{r} \gamma_{i j}=\sum_{j=1}^{s} \gamma_{i j}=0, \\
\varepsilon_{i j k} \sim N\left(0, \delta^{2}\right) i=1, \cdots, r, j=1, \cdots, s, k=1, \cdots, t
\end{array}\right.
$$

Original hypothesis is:

$$
\begin{aligned}
& H_{01}: \alpha_{i}=0(i=1, \cdots, r) \\
& H_{02}: \beta_{j}=0(j=1, \cdots, s) \\
& H_{03}: \gamma_{i j}=0(i=1, \cdots, r, j=1, \cdots, s)
\end{aligned}
$$

If two factors have no interaction effects, let $t=1$, process can simplify, assume $\gamma_{i j}=0$, then:

$\mu_{i j}=\mu+\alpha_{i}+\beta_{i}, i=1, \cdots, r, j=1, \cdots, s$

Now, model can be written as:

$$
\left\{\begin{array}{l}
x_{i j}=\mu+\alpha_{i}+\beta_{j}+\varepsilon i_{j} \\
\sum_{i=1}^{r} \alpha_{i}=0, \sum_{j=1}^{s} \beta_{j}=0 \\
\varepsilon_{i j k} \sim N\left(0, \delta^{2}\right) i=1, \cdots, r, j=1, \cdots, s
\end{array}\right.
$$

Below is test statistics:

$$
\begin{aligned}
& \bar{x}=\frac{1}{r s} \sum_{i=1}^{r} \sum_{j=1}^{s} x_{i j}, x_{i \bullet}=\frac{1}{s} \sum_{j=1}^{s} x_{i j}, x_{\bullet j}=\frac{1}{r} \sum_{i=1}^{r} x_{i j} \\
& S_{T}=\sum_{i=1}^{r} \sum_{j=1}^{s}\left(x_{i j}-\bar{x}\right)^{2}
\end{aligned}
$$

Among them, $S_{T}$ is whole test data headquarter variation, and becomes total squares sum, decompose it:

Table 2. Variance analysis data table.

\begin{tabular}{|c|c|c|c|c|}
\hline & $B_{1}$ & $B_{2}$ & $\cdots$ & $B_{s}$ \\
\hline \hline$A_{1}$ & $x_{111} \cdots x_{11 t}$ & $x_{121} \cdots x_{12 t}$ & $\cdots$ & $x_{1 s 1} \cdots x_{1 s t}$ \\
\hline$A_{2}$ & $x_{211} \cdots x_{21 t}$ & $x_{221} \cdots x_{22 t}$ & $\cdots$ & $x_{2 s 1} \cdots x_{2 s t}$ \\
\hline$\vdots$ & $\vdots$ & $\vdots$ & $\vdots$ & $\vdots$ \\
\hline$A_{r}$ & $x_{r 11} \cdots x_{r 1 t}$ & $x_{r 21} \cdots x_{r 2 t}$ & $\cdots$ & $x_{r s 1} \cdots x_{r s t}$ \\
\hline
\end{tabular}


Table 3. Variance analysis result.

\begin{tabular}{|c|c|c|c|}
\hline & Item & City $M \pm S D$ & Countryside $M \pm S D$ \\
\hline \multirow{4}{*}{ Sports forms participation } & The network, the library & $0.43 \pm 0.15$ & $0.11 \pm 0.08$ \\
\hline & Associations, clubs & $0.47 \pm 0.08$ & $0.07 \pm 0.07$ \\
\hline & Experts, social instructors & $0.33 \pm 0.24$ & $0.13 \pm 0.19$ \\
\hline & Friends, colleagues & $0.67 \pm 0.11$ & $0.63 \pm 0.13$ \\
\hline \multirow{3}{*}{ Sports activities organizations } & The government sector organizations & $0.2398 \pm 0.35$ & $0.3333 \pm 0.28$ \\
\hline & Associations of social organizations & $0.4122 \pm 0.14$ & $0.4608 \pm 0.10$ \\
\hline & Social sponsorship & $0.348 \pm 0.21$ & $0.2062 \pm 0.17$ \\
\hline \multirow{4}{*}{ Sports consciousness } & Manual work instead of physical fitness & $0.02 \pm 0.17$ & $0.12 \pm 0.09$ \\
\hline & No disease or health & $0.05 \pm 0.20$ & $0.47 \pm 0.11$ \\
\hline & Physical fitness is equal to the waste of time & $0.03 \pm 0.22$ & $0.21 \pm 0.18$ \\
\hline & Fitness to promote physical and mental health & $0.90 \pm 0.11$ & $0.20 \pm 0.05$ \\
\hline \multirow{4}{*}{ Public satisfaction index } & Satisfaction & $0.10 \pm 0.09$ & $0.04 \pm 0.03$ \\
\hline & Basic satisfaction & $0.40 \pm 0.04$ & $0.16 \pm 0.01$ \\
\hline & Dissatisfaction & $0.45 \pm 0.21$ & $0.61 \pm 0.20$ \\
\hline & Does not matter & $0.05 \pm 0.19$ & $0.19 \pm 0.11$ \\
\hline
\end{tabular}

$$
\begin{aligned}
S_{T} & =\sum_{i=1}^{r} \sum_{j=1}^{s}\left(x_{i j}-\bar{x}\right)^{2} \\
& =\sum_{i=1}^{r} \sum_{j=1}^{s}\left(x_{i j}-\overline{x_{i \bullet}}-\bar{x} \bullet j+\bar{x}\right)^{2}+s \sum_{i=1}^{r}\left(x_{i i \bullet}-\bar{x}\right)^{2}+r \sum_{j=1}^{s}\left(x_{\bullet j}-\bar{x}\right)^{2} \\
& =S_{E}+S_{A}+S_{B}
\end{aligned}
$$

It can verify: in above squares sum decomposition, cross terms are 0 .Among them:

$$
\begin{aligned}
& S_{E}=\sum_{i=1}^{r} \sum_{j=1}^{s}\left(x_{i j}-\bar{x}_{i \bullet}-\bar{x}{ }_{\bullet j}+\bar{x}\right)^{2} \\
& S_{A}=s \sum_{i=1}^{r}\left(x_{i i \bullet}-\bar{x}\right)^{2} \\
& S_{B}=r \sum_{j=1}^{s}\left(x_{\bullet j}-\bar{x}\right)^{2}
\end{aligned}
$$

When $H_{01}$ is true:

$$
F_{A}=\frac{\frac{S_{A}}{r-1}}{\frac{S_{E}}{(r-1)(s-1)}} \sim F(r-1,(r-1)(s-1))
$$

When $H_{02}$ is true:

$$
F_{B}=\frac{\frac{S_{B}}{r-1}}{\frac{S_{E}}{(r-1)(s-1)}} \sim F(s-1,(r-1)(s-1))
$$

Test rules are:

When $\quad F_{A}<F_{1-a}(r-1,(r-1)(s-1)), \quad$ accept $\quad H_{01}$, otherwise refuse $H_{01}$;

When $\quad F_{B}<F_{1-a}(s-1,(r-1)(s-1)), \quad$ accept $\quad H_{02}$, otherwise refuse $H_{02}$.

\subsection{Data Analysis}

Use MATLAB software to analyze Table 1, and then get following variance analysis data Table 3 .

By above variance analysis data table, it can get conclusion that urban sports and rural sports have obvious differences in residents' participation in sports activities forms, urban and rural sports interactive organizations, urban and rural residents' sports consciousness and urban and rural residents' sports satisfaction index aspect, the difference size can be presented by above data table numerical values. Among them, in data table, data is the reflection of mean value and variance after variance analyzing, variance gets bigger, and then it shows the two differences to be bigger.

\section{URBAN SPORTS DEVELOPMENT AND URBANI- ZATION CONSTRUCTION CORRELATION ANALY-} SIS

Correlation analysis is utilizing software to handle with data, comparing correlation by comparing correlation coefficient size. Common correlation coefficient has Pearson correlation coefficient and Spearman rank correlation coefficient. The paper utilizes Pearson correlation coefficient to make correlation comparison. 
Table 4. Correlation data table.

\begin{tabular}{|c|c|c|}
\hline & & Country Side \\
\hline \multirow{4}{*}{ City } & Pearson correlation & .240 \\
\hline & Significance (bilateral) & .388 \\
\hline & Square and cross product sum & .164 \\
\hline & Covariance & .012 \\
\hline \multicolumn{3}{|c|}{ Significant correlated in 0.01 level } \\
\hline
\end{tabular}

Pearson correlation coefficient is used to show two variables similarity extent mathematical statistical quantity, it can be used to make quantitative calculation on two variables similarities. Its calculation formula is as following:

$$
\rho(X, Y)=\frac{\operatorname{cov}(X, Y)}{\sigma_{x} \sigma_{y}}=\frac{E\left(\left(X-\mu_{x}\right)\left(Y-\mu_{y}\right)\right)}{\sigma_{x} \sigma_{y}}
$$

Among them, covariance is used as numerator, is product of two variables standard deviation, and it requires two variables standard deviation not to be 0 .

$$
\text { And } \mu_{x}=E(X), \sigma_{X}{ }^{2}=E\left(X-\mu_{x}\right)^{2}=E\left(X^{2}\right)-E^{2}(X)
$$

Therefore, Pearson correlation coefficient can also be written as:

$$
\rho(X, Y)=\frac{E(X Y)-E(X) E(Y)}{\sqrt{E\left(X^{2}\right)-E^{2}(X)} \sqrt{E\left(Y^{2}\right)-E^{2}(Y)}}
$$

When two variables Pearson correlation coefficient gets closer to 1 or -1 , it shows the two correlation is big, or closely related. It gets closer to 1 shows the two are in positive correlation, on the contrary it gets closer to -1 shows the two are in negative correlation.

\subsection{Data Processing}

For data Table 1, carry out correlation analysis, analyze rural sports and urban sports correlation sizes in residents participation sports activities forms, urban and rural sports interactive organizations, urban and rural residents' sports consciousness and urban and rural residents' sports satisfaction index aspects, and then research on rural sports and urban sports impacts on urbanization construction, result is as Table 4.

By above analysis, it can get conclusion: urban sports is closely related to rural sports construction, only urban sports drives rural sports then can propel to rural sports development, and only rural sports develops then can let urban and rural gap to be smaller and promote urbanization construction progress.

\section{CONCLUSION}

The paper firstly compares Chinese urban and rural sports development, it carries out comprehensive evaluation on urban and rural residents' sports activities participation forms, urban and rural sports interactive organization, urban and rural residents' sport consciousness and urban and rural residents' sports satisfaction index, finds out urban and rural sports development gap, and utilizes software to process with data, by statistical figure, it more intuitive gets conclusions: urban sports development is faster, no matter urban residents' sports consciousness, or in the aspect of sports activities participation, all are better than rural residents, its data gap is larger. In addition, on the basis of urban and rural sports development basic information researching, utilize variance analysis and correlation analysis; it further researches on urban and rural sports development difference significance. Among them, by variance analysis method to make analysis of urban and rural sports residents participation sports activities forms, urban and rural sports interactive organizations, urban and rural residents sports consciousness and urban and rural residents' sports satisfaction index each kind of indicator data, it gets conclusions. Secondly, by correlation analysis, establish urban sports and rural sports development correlation model, and then compare correlation coefficient sizes, finally get conclusions: both urban sports and rural sports have significant differences in each aspect construction, the two are closely linked. Rural sports restricts urban sports development, urban sports drive rural sports development, only the two make joint advancement, shorten gap, then can construct better urbanization life environment.

\section{CONFLICT OF INTEREST}

The authors confirm that this article content has no conflict of interest.

\section{ACKNOWLEDGEMENTS}

Declared none.

\section{REFERENCES}

[1] C. Po, Q. Zhong-Mei, Y. Ying, and X. Chong-De, "Correlation analysis of current situation of regional athletics sports development and society population structure in china," J. Beijing. Sport. Univ., vol. 30, no. 12, pp. 1610-1613, 2007.

[2] G. Hong, "Summary on chinese sports population research since 1980s,” China. Sport. Sci. Technol., vol. 43, no. 3, pp. 36-40, 2007.

[3] L. Hong, X. Hai-hong, and F. Wu-long, "Sociological analysis of comparison of chinese population with the sports population of chinese," J. Xi'an. Inst. Phys Educ., vol. 24, no. 4, pp. 25-28, 2007.

[4] M. Zhi-wen, and Q. Chun-lin, "Sociological analysis of contemporary Chinese sports population structures," J. Phys. Educ., vol. 13, no. 1, pp. 119-121, 2006.

[5] X. Huan-yu, W. Zhi-qiang, and C. Yu-zhong, "Basic characteristics of social and sports population structures of contemporary china," J. Shanghai. Phys Educ. Inst., vol. 29, no. 2, pp. 10-14, 2005. 
[6] X. Jin-xia, and W. Jing-tong, "Analysis on development level of competitive sports of eastern china in the "eleventh five-year" Period," Bull. Sport. Sci. Technol., vol. 19, no. 4, pp. 57-59, 2011.
[7] Y. De-yi, "Development of sports for all under circumstance of building well-off society," J. Wuhan. Inst. Phys. Educ., vol. 40, no. 1, pp. 15-19, 2006.

Received: June 10, 2015

Revised: July 29, 2015

Accepted: August 15, 2015

(C) Wang et al.; Licensee Bentham Open.

This is an open access article licensed under the terms of the (https://creativecommons.org/licenses/by/4.0/legalcode), which permits unrestricted, noncommercial use, distribution and reproduction in any medium, provided the work is properly cited. 